\title{
Cryptanalysis of Improved Biometric-Based User Authentication Scheme for C/S System
}

\author{
Younsung Choi, Donghoon Lee, Jiye Kim, Jaewook Jung, and Dongho Won
}

\begin{abstract}
Password-based authentication schemes are convenient, but vulnerable to simple dictionary attacks. Cryptographic secret keys are safe, but difficult to memorize. Recently, biometric information has been used for authentication schemes because it is difficult to copy, share, forge, and distribute. In 2011, Das proposed a biometric-based authentication scheme, but it has various vulnerabilities such as replay attack, denial-of-service attack, user impersonation attack, and password change problem. To solve these problem, Jiping et al. improved Das's scheme, but some vulnerabilities have remained still. In this paper, we analyze the cryptanalysis of Jiping et al. authentication scheme. So this paper shows that Jiping et al.'s scheme is vulnerable against server masquerading attack and stolen smart card attack. And also we show the possibility of authentication without login phase in Jiping et al.'s authentication scheme.
\end{abstract}

Index Terms - User authentication, biometric-base scheme, client/server system, cryptanalysis on authentication scheme.

\section{INTRODUCTION}

Remote identity-based authentication schemes have been based primarily on passwords alone. Password-based authentication schemes provide a simple and convenient method to authenticate users before providing them with the services of a computing or communication system [1]-[7]. However, the use of passwords alone is a scheme that is easy to break by using simple dictionary attacks. To overcome this problem, cryptographic secret keys and passwords have been used in remote-user authentication schemes [8]-[10]. Long and random cryptographic keys are difficult to memorize, however, so they must be stored somewhere, which is a weak point. To solve this problem, various biometric-based authentication schemes have been proposed. Das proposed a new biometric-based authentication scheme, but it has various vulnerabilities [11]. Subsequently, Jiping et al. proposed a security-improved biometric-based user authentication scheme for $\mathrm{C} / \mathrm{S}$ system based on Das's authentication scheme [12], but Jiping et al.'s scheme has some remaining the security problems.

The rest of this paper is organized as follows. In Section II, we review related works. In Section III, we briefly review Jiping et al.'s biometric-based remote user authentication scheme using smart cards. In Section IV, we analyze the security vulnerability of Jiping et al.'s scheme and suggest a solution. Finally, we conclude the paper in Section V.

Manuscript received April 5, 2014; revised June 5, 2014

The authors are with the School of Information and Communication Engineering, Sungkyunkwan University, Korea (e-mail: yschoi@security.re.kr, dhlee@security.re.kr, jykim@security.re.kr, jwjung@security.re.kr, dhwon@security.re.kr).

\section{RELATED WORKS}

\section{A. Smart Card Attack}

Various researchers have observed that the confidential information stored in all smart cards could be extracted by physically monitoring power consumption, such as by using SPA and DPA. When a user loses a smart card, an attacker can analyze it. Various schemes leave it vulnerable to off-line password attacks in which an attacker can be authenticated to the server without having the user's ID and password.

\section{B. Biometric-Based Authentication}

Biometrics refers to the quantifiable data related to human characteristics and traits. Examples include fingerprints, face recognition, DNA, palm prints, hand geometry, iris, retina, odor/scent, typing rhythm, gait, and voice. Biometrics-based authentication can be used in user identification and access control. Biometric information cannot be lost or forgotten and is very difficult to copy, share, forge or distribute. In addition, biometric information cannot be guessed easily and such coding is more difficult to break than other types.

\section{Das's Biometric-Based User Authentication Scheme}

Das proposed biometric-based remote user authentication, which is inherently more reliable and secure than traditional password-based remote authentication schemes. However, this scheme has security vulnerabilities to replay attack, denial-of-service attack, user impersonation attack, and password change problems. Moreover, this scheme does not provide mutual authentication between the user and the server.

\section{REVIEW OF JIPING ET AL.'S SCHEME}

TABLE I: NOTATION

\begin{tabular}{c|c}
\hline $\begin{array}{c}\text { Notatio } \\
\mathrm{n}\end{array}$ & Description \\
\hline \hline$C_{i}$ & Client \\
$S_{i}$ & Server \\
$R_{i}$ & Registration center \\
$P W_{i}$ & Password shared between $C_{i}$ and $S_{i}$ \\
$I D_{i}$ & Identity of the user $C_{i}$ \\
$B_{i}$ & Biometric template of the user $C_{i}$ \\
$d(\cdot)$ & Symmetric parametric function \\
$\tau$ & Predetermined threshold \\
$h(\cdot)$ & A secure one-way hash function \\
$X_{s}$ & A secret information maintained by the server \\
$R_{c}$ & A random number chosen by $C_{i}$ \\
$R_{s}$ & A random number chosen by $S_{i}$ \\
$A \| B$ & Data $A$ concatenates with data $B$ \\
$A \oplus B$ & XOR operation of $A$ and $B$ \\
\hline \hline
\end{tabular}


Das proposed a biometric-based authentication scheme that has various security problems. To solve these problems, Jiping et al. proposed an improved biometric-based authentication scheme [12]. In the following review of Jiping et al.'s scheme, for convenience, we use the notations shown in Table I.

\section{A. Registration Phase}

In the registration phase, remote user $C_{i}$ must perform the following registration steps, as shown in Fig. 1 for the registration phase of Jiping's scheme.

\begin{tabular}{ll} 
Client $\left(C_{i}\right)$ & Registration Center $\left(R_{i}\right)$ \\
\hline
\end{tabular}

(1) chooses $I D_{i}, B_{i}, P W_{i}$

$\left\langle I D_{i}, B_{i}, P W_{i}\right\rangle$
$(2)$ computes $f_{i}, g_{i}, r_{i}$ and $e_{i}$
$f_{i}=h\left(B_{i}\right)$
$g_{i}=h\left(I D_{i}\right)$
$r_{i}=h\left(P W_{i}\right) \oplus f_{i}$
$e_{i}=h\left(g_{i} \| X_{s}\right) \oplus r_{i}$

(3) receives smart card

Fig. 1. Registration phase of Jiping et al.'s scheme.

1) The user $C_{i}$ inputs personal biometric $B_{i}$ on a device and sends the identity $\mathrm{ID}_{i}$ and password $\mathrm{PW}_{i}$ to the registration center $R_{i}$ in person.

2) The registration center $R_{i}$ computes $f_{i}=h\left(B_{i}\right), g_{i}=$ $h\left(\mathrm{ID}_{i}\right), r_{i}=h\left(\mathrm{PW}_{i}\right) \oplus f_{i}$, and $e_{i}=h\left(g_{i} \| X_{s}\right) \oplus r_{i} . X_{s}$ is secret information shared by $R_{i}$ and $S_{i} . X_{S}$ and the passwords of the corresponding users are not disclosed to any others for all future secure communications.

3) Registration center $R_{i}$ loads $\left(h(\cdot), f_{i}, g_{i}, e_{i}, r_{i}, \tau, d(\cdot)\right)$ on the user's smart card and sends this information to user $C_{i}$ using a secure channel.

\section{B. Login Phase}

In the login phase, remote user $C_{i}$ must perform the following login steps, as shown in Fig. 2.

$$
\text { Client }\left(C_{i}\right)
$$$$
\text { Server }\left(S_{i}\right)
$$

(1) inserts the smart card and $B_{i}^{\prime}$

(2) verifies whether $d\left(B_{i}, B_{i}^{\prime}\right)<\tau$ ?

(3) if it holds, then $C_{i}$ inputs his/her password $P W_{i}$

(4) computes $r_{i}^{\prime}=h\left(P W_{i}\right) \oplus f_{i}$ and verifies whether $d\left(r_{i}, r_{i}^{\prime}\right)<\tau$ ?

(5) if it holds, the smart card computes the following:

$$
M_{1}=e_{i} \oplus r_{i}^{\prime}
$$

$M_{2}=h\left(R_{c} \| T\right)$

$M_{3}=M_{1} \oplus M_{2} \longrightarrow\left\langle g_{i}, M_{2}, M_{3}, T\right\rangle$

(7)

Fig. 2. Login phase of Jiping et al.'s scheme.

1) $C_{i}$ first inserts the user's smart card into the card reader of a terminal and inputs the user's biometric template $B^{\prime}{ }_{i}$ on the device. If $d\left(B_{i}, B_{i}^{\prime}\right)>\tau$, the login phase is terminated. Otherwise, $C_{i}$ passes the biometric verification and then inputs the user's password $\mathrm{PW}_{i}$.

2) The smart card computes $r_{i}^{\prime}=h\left(\mathrm{PW}_{i}\right) \oplus f_{i}$. If $d\left(r_{i}^{\prime}, r_{i}\right)>$ $\tau$, then the password is not correct, so the system terminates the session; otherwise, the smart card computes $M_{1}=e_{i} \oplus r_{i}^{\prime}$, which is equal to $h\left(g_{i} \| X_{S}\right), M_{2}$ $=h\left(R_{c} \| T\right)$, where $R_{c}$ is a random number generated by the user $C i, T$ is the current timestamp of $C_{i}$ 's system, and $M_{3}=M_{1} \oplus M_{2}$.

3) Finally, user $C_{i}$ sends the message $\left\langle g_{i}, M_{2}, M_{3}, T\right\rangle$ to the remote server $S_{i}$.

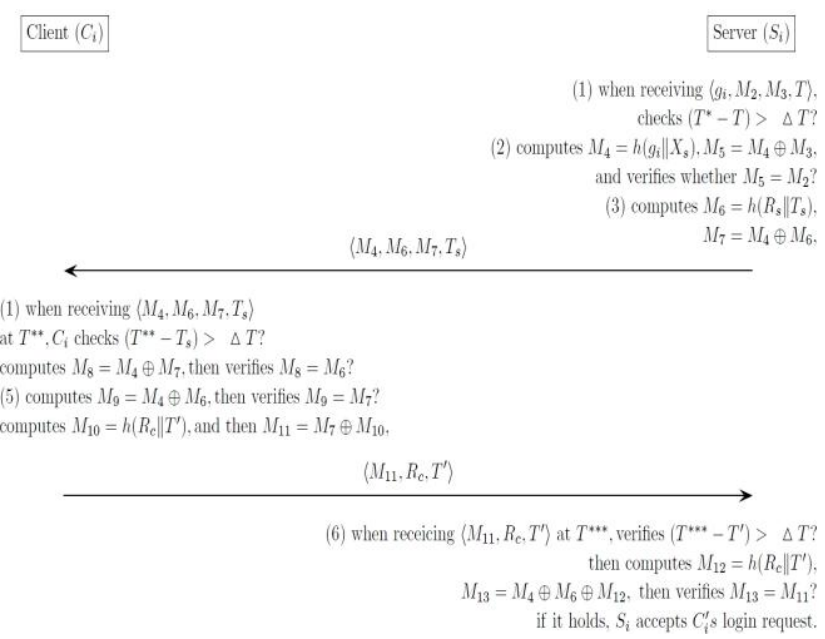

Fig. 3. Authentication phase of Jiping et al.'s scheme.

\section{Authentication Phase}

In the authentication phase, remote user $C_{i}$ and server $S_{i}$ must perform the following authentication phase. When the remote server $S_{i}$ receives the login message $\left\langle g_{i}, M_{2}, M_{3}, T\right\rangle$ at time $T^{*}$, it will perform the following steps, as shown in Fig. 3, to authenticate whether the user $C_{i}$ is legitimate.

1) Check $T$. If $\left(T^{*}-T\right)>\Delta T$, the authentication phase is terminated, where $\Delta T$ is the expected time interval for the transmission delay of the system. Otherwise, if $\left(T^{*}\right.$ $T) \leq \Delta T$, the authentication steps will be performed.

2) $S_{i}$ checks the accuracy of $C_{i}$ 's ID ID $_{i}$ It computes $M_{4}=h\left(g_{i}\right.$

$\| X_{s}$ ) using the secret value $X_{s}$ maintained by the server $S_{i}$ and then computes $M_{5}=M_{4} \oplus M_{3}$ and verifies whether $M_{5}=M_{2}$ or not. If it is not accurate, then $S_{i}$ rejects $C_{i}$ 's login request. If the verification is successful, the next step will be performed.

3) $S_{i}$ computes $M_{6}=h\left(R_{s} \| T_{s}\right)$ and $M_{7}=M_{4} \oplus M_{6}$, where $T_{s}$ is the timestamp of the server $S_{i}$, and then $S_{i}$ sends message $\left\langle M_{4}, M_{6}, M_{7}, T_{s}\right\rangle$ to user $C_{i}$.

4) After receiving the message $\left\langle M_{4}, M_{6}, M_{7}, T_{s}\right\rangle$ at time $T^{* *}$, $C_{i}$ checks the freshness of $T_{s}$ by verifying $\left(T^{* *}-T_{s}\right)>$ $\Delta T$. If it holds, the following session is terminated; otherwise $C_{i}$ computes $M_{8}=M_{4} \oplus M_{7}$ and then verifies whether $M_{8}=M_{6}$. If it does not hold, $C_{i}$ terminates the session. Otherwise, it goes to the next step.

5) $C_{i}$ computes $M_{9}=M_{4} \oplus M_{6}$ and then verifies whether $M_{9}$ $=M_{7}$. If it does not hold, $S_{i}$ is rejected by $C_{i}$; otherwise, if it holds, $C_{i}$ computes $M_{10}=h\left(R_{c} \| T^{\prime}\right)$, where $T^{\prime}$ is the current timestamp of user $C_{i}$, and then computes $M_{11}=$ $M_{7} \oplus M_{10}$ and sends the message $\left\langle M_{11}, R_{c}, T^{\prime}\right\rangle$ to the remote server $S_{i}$.

6) Step 6: When $S_{i}$ receives the message $\left\langle M_{11}, R_{c}, T^{\prime}\right\rangle$ at time $T^{* * *}$, it checks $\left(T^{* * *}-T^{\prime}\right)>\Delta T$. If it holds, the authentication phase is terminated. Otherwise, if it does not hold, $S i$ computes $M_{12}=\mathrm{h}\left(R_{c} \| T^{\prime}\right)$ and then computes $M_{13}=M_{4} \oplus M_{6} \oplus M_{12}$. After computing $M_{13}$, then $S_{i}$ 
verifies whether $M_{13}=M_{11}$. If it holds, $S_{i}$ accepts $C_{i}$ 's login request; otherwise, $S_{i}$ rejects the login request.

\section{Password Change Phase}

In Jiping et al.'s scheme, user $C_{i}$ can freely change the password $\mathrm{PW}_{\text {old } i}$ to a new one $\mathrm{PW}_{\text {newi }}$. The password change procedure is performed as follows.

1) $C_{i}$ inserts the smart card into the card reader and offers the user's personal biometrics $B^{\prime}{ }_{i}$. The smart card computes $f^{\prime} i=h\left(B_{i}^{\prime}\right)$ and verifies it by checking $d\left(f^{\prime}{ }_{i}\right.$, $\left.f_{i}\right) \leq \tau$. Where $f_{i}=h\left(B_{i}\right)$ is the information stored in the smart card.

2) If it holds, $C_{i}$ inserts old password $\mathrm{PW}_{\text {old } i}$ and new password $\mathrm{P}_{\text {Wnewi. }}$. Otherwise, the password change procedure is terminated.

3) The smart card performs $r_{i}^{\prime}=h\left(\mathrm{PW}_{\text {old } i}\right) \oplus{f^{\prime}}_{i}$ and checks $d\left(r_{i}^{\prime}, r_{i}\right) \leq \tau . r_{i}$ is the information stored in the smart card.

4) If it holds, the smart card computes $r_{i}^{\prime}=h\left(\mathrm{PW}_{\text {new } i}\right) \oplus f_{i}$, $e^{\prime}{ }_{i}=e_{i} \oplus r_{i}\left(=h\left(\operatorname{ID}_{i} \| X_{S}\right)\right)$, and $e^{\prime \prime}{ }_{i}=e^{\prime}{ }_{i} \oplus r_{i}$.

5) Finally, replace $e_{i}$ with $\mathrm{e}^{\prime \prime}{ }_{i}$ and $r_{i}$ with $r^{\prime \prime}{ }_{i}$ on smart card.

\section{CRYPTANALYSIS OF JiPING ET AL.'S SCHEME}

Jiping et al. enhanced the security of Das's authentication scheme and proposed a new authentication scheme. But Jiping et al.'s authentication scheme has some remaining the security problems of vulnerabilities to the server masquerading attack, stolen smart-card attack, and authentication without login phase.

\section{A. Server Masquerading Attack}

An attacker can masquerade as a legitimate server if the attacker knows $h\left(g_{i} \| X_{s}\right)$. For this reason, the server authenticates the user using only $h\left(g_{i} \| X_{s}\right)$. Fig. 4 shows the phases of a server-masquerading attack. First, the attacker intercepts the client's message $\left\langle g_{i}, M_{2}, M_{3}, T>\right.$. Then, the attacker calculates $h\left(g_{i} \| X_{s}\right)$ using $\mathrm{M}_{2} \oplus \mathrm{M}_{3}$. Because $h\left(g_{i} \|\right.$ $\left.X_{s}\right)=e_{i} \oplus r_{i}=M_{2} \oplus M_{3}$, the attacker computes $M_{4}, M_{6}, M_{7}$ and $T_{A}$ using $h\left(g_{i} \| X_{S}\right)$ and sends them to the client. The client checks and authenticates messages. The attacker receives $M_{11}$, $R_{c}$, and $T^{\prime}$ and then checks whether the attacker is successful at masquerading as the server. The client is authenticated with the attacker. In Jiping et al.'s scheme, the attacker can execute the server-masquerading attack. To solve this problem, it is necessary to add another piece of security information to authenticate the client with the server, such that the attacker cannot compute the security information using messages communicated between the server and the client.

\section{B. Stolen Smart Card Attack}

Kocher et al. and Messerges et al. pointed out that the confidential information stored in all existing smart cards could be extracted by physically monitoring their power consumption. Therefore, if a user loses a smart card, all private information in the smart card could be revealed to an attacker [13]-[15]. In Jiping et al.'s scheme, a smart card stores various secrets for the login and authentication of the user.

$$
\begin{aligned}
& \text { Client }\left(C_{i}\right) \\
& M_{1}=e_{i} \oplus r_{i}^{\prime} \\
& M_{2}=h\left(R_{c} \| T\right) \\
& M_{3}=M_{1} \oplus M_{2}
\end{aligned}
$$

Attacker $\left(A_{i}\right)$

(1) $\mathrm{A}_{\mathrm{i}}$ intercept client's message $\left\langle g_{i}, M_{2}, M_{3}, T\right\rangle$

(2) $\mathrm{A}_{\mathbf{i}}$ calculates $h\left(g_{i} \| X_{s}\right)=e_{i} \oplus r_{i}^{\prime}=M_{2} \oplus M_{3}$

(3) $A_{i}$ masquerades as server

(4) computes $M_{4}=h\left(g_{i} \| X_{s}\right)$

(5) computes $M_{6}=h\left(R_{A} \| T_{A}\right)$

(6) computes $M_{7}=M_{4} \oplus M_{6}$

(7) $\mathrm{A}_{\mathbf{i}}$ send message to $\mathrm{C}_{\mathbf{i}}\left\langle M_{4}, M_{6}, M_{7}, T_{A}\right\rangle$

(8) when receiving $\left\langle M_{4}, M_{6}, M_{7}, T_{A}\right\rangle$

at $T^{* *}, C_{i}$ checks $\left(T^{* *}-T_{A}\right)>\triangle T$ ?

computes $M_{8}=M_{4} \oplus M_{7}$, then verifies $M_{8}=M_{6}$ ?

(9) computes $M_{9}=M_{4} \oplus M_{6}$, then verifies $M_{9}=M_{7}$ ?

computes $M_{10}=h\left(R_{A} \| T^{\prime}\right)$, and then $M_{11}=M_{7} \oplus M_{10}$

$$
\begin{array}{r}
\left\langle M_{11}, R_{A}, T^{\prime}\right\rangle \\
(10) \text { when } \mathrm{A}_{\mathrm{i}} \text { receives }\left\langle M_{11}, R_{A}, T^{\prime}\right\rangle \text { at, then } \mathrm{A}_{\mathrm{i}} \text { computes } M_{12}=h\left(R_{A} \| T^{\prime}\right) \\
M_{13}=M_{4} \oplus M_{6} \oplus M_{12}, \text { then verifies } M_{13}=M_{11} \text { ? }
\end{array}
$$

(11) if it corrects, $A_{i}$ success to masquerades as server

Fig. 4. Server masquerading attack.

The smart card for user $\mathrm{ID}_{\mathrm{i}}$ includes $\left(h(\cdot), f_{i}, g_{i}, e_{i}, r_{i}, \tau\right.$, $d(\cdot))$. So, if an attacker obtains the user's smart card, the attacker can acquire the user's $f_{i}, g_{i}$, and $r_{i}$. The attacker can calculate $h\left(P W_{i}\right)$ and $h\left(I D_{i}\right)$, then execute an off-line password attack using the rainbow table, dictionary attack, and brute attack to obtain $I D_{i}$ and $P W_{i}$. For this reason, the $I D_{i}$ and $P W_{i}$ are protected using $h(\cdot)$. To solve this problem, it is necessary to add a random number with high-entropy. Fig. 5 shows the phases of a stolen smart card attack.

\section{Authentication without Login Phase}

In Jiping et al.'s scheme, attacker can be authenticate with server without login phase. To skip the login phase, the attacker need to still or get the user's smart card. In other words, if the attacker obtain user's smart card, the attacker can be authenticate to server without user's $I D_{i}, P W_{i}$ and 
user's biometric information $B_{i}$. Fig. 6 shows the phase of authentication without login phase.

\section{Attacker}

gets(steals) user's smart card

obtains information from smart card using SPA and DPA

$$
\rightarrow \operatorname{gets} h(\cdot), f_{i}, g_{i}, e_{i}, r_{i}, \tau \text { and } d(\cdot)
$$

Attacker knows $f_{i}, g_{i}, r_{i}$

$$
\begin{aligned}
r_{i} & =h\left(P W_{i}\right) \oplus f_{i} \\
& \rightarrow h\left(I D_{i}\right)=g_{i} \\
& \rightarrow h\left(P W_{i}\right)=r_{i} \oplus f_{i}
\end{aligned}
$$

executes off-line password attack

$\rightarrow$ figures out user's ID and password $I D_{i}, P W_{i}$ Fig. 5. Stolen smart-card attack.

Firstly, attacker gets or steals the user's smart card and obtains information from smart card using SPA and DPA. So the attacker can generate and compute the $R_{c}, M_{1}, M_{2}$ and $M_{3}$ using this information. And the attacker sends $\left\langle g_{i}, M_{2}, M_{3}, T\right\rangle$ to the server. Then, the attacker receives $\left\langle M_{4}, M_{6}, M_{7}, T_{s}\right\rangle$ and then, the attacker can computes $\left\langle M_{11}, R_{c}, T^{\prime}\right\rangle$ and send these messages to server. So attacker can be authenticated to the server without user's $I D_{i}, P W_{i}$ and the user's biometric information $B_{i}$. To solve this problem, it is necessary to add information of user's $P W_{i}$ or $B_{i}$ to authentication messages.

\section{Attacker}

gets(steals) user's smart card

obtains information from smart card using SPA and DPA

$$
\rightarrow \operatorname{gets} h(\cdot), f_{i}, g_{i}, e_{i}, r_{i}, \tau \text { and } d(\cdot)
$$

computes $M_{1}, M_{2}, M_{3}$

$\rightarrow$ generates random number $R_{c}$

$\rightarrow M_{1}=e_{i} \oplus r_{i}$

$\rightarrow M_{2}=h\left(R_{c} \| T\right)$

$\rightarrow M_{3}=M_{1} \oplus M_{2}$

sends login and authentication message to $S_{i}$

$\rightarrow\left\langle g_{i}, M_{2}, M_{3}, T\right\rangle$

receives $S_{i}^{\prime} s$ message

$$
\rightarrow\left\langle M_{4}, M_{6}, M_{7}, T_{s}\right\rangle
$$

computes $M_{11}, R_{c}, T^{\prime}$

$\rightarrow$ generates timestamp $T^{\prime}$

$$
\rightarrow M_{10}=h\left(R_{c} \| T^{\prime}\right)
$$$$
\rightarrow M_{11}=M_{8} \oplus M_{10}
$$

sends authentication message to $S_{i}$

$\rightarrow\left\langle M_{11}, R_{c}, T^{\prime}\right\rangle$

$\rightarrow$ attacker can be authenticated with $S_{i}$

Fig. 6. Authentication without login phase.

\section{CONCLUSION}

In this paper, we analyze the cryptanalysis of Jiping et al.'s biometric-based user authentication scheme for the client/server system. Jiping et al. proposed an improved authentication scheme to solve the problem of vulnerabilities in Das's scheme. However, Jiping et al.'s scheme has some remaining security problems: the server-masquerading attack, stolen smart-card attack and authentication without login phase. To solve this problem, it is necessary to add secret information to the registration, login and authentication phases.

\section{ACKNOWLEDGMENTS}

This research was supported by the MSIP(Ministry of Science, ICT and Future Planning), Korea, under the ITRC(Information Technology Research Center) support program (NIPA-2014-H0301-14-1004) supervised by the NIPA(National IT Industry Promotion Agency)

\section{REFERENCES}

[1] C. T. Li, "An enhanced remote user authentication scheme providing mutual authentication and key agreement with Smart Cards," in Proc. the 5th International IEEE Computer Society Conference on Information Assurance and Security, Xi'an, China, 2009, pp. 517-520.

[2] K. H. M. Wong, Z. Yuan, C. Jiannong, and W. Shengwei, "A dynamic user authentication scheme for wireless sensor networks," in Proc. the IEEE International Conference on Sensor Networks, Ubiquitous, and Trustworthy Computing (SUTC '06), Taichung, Taiwan, June 2006, pp. 244-251.

[3] T. H. Lee, "Simple dynamic user authentication protocols for wireless sensor networks," in Proc. the 2nd International Conference on Sensor Technologies and Application, CapEsterel, France, August 2008, pp. 657-660.

[4] B. Vaidya, J. J. Rodrigues, and J. H. Park, "User authentication schemes with pseudonymity for ubiquitous sensor network in NGN," International Journal of Communication Systems, vol. 23, no. 9-10, pp. 1201-1222, 2010.

[5] N.Y. Lee and Y. C. Chiu, "Improved remote authentication scheme with smart card," Computer Standards and Interfaces, vol. 27, no. 2, pp. 177-180, 2005.

[6] M. Kim and C. K. Koc, "Asimple attackon a recently introduced hash-based strong-password authentication scheme," International Journal of Network Security, vol. 1, no. 2, pp. 77-80, 2005.

[7] L. C. Ko, "A novel dynamic user authentication scheme for wireless sensor networks," in Proc. the IEEE International Symposium on Wireless Communication Systems, Reykjavik, Iceland, October 2008, pp. 608-612.

[8] J. Nam, K. K. R. Choo, M. Kim, J. Paik and D. Won, "Dictionary Attacks against Password-Based Authenticated Three- Party Key exchange protocols," KSII Transactions on Internet and Information Systems, vol. 7, no. 12, December 2013, pp. 3244-3260.

[9] J. Nam, K. K. R. Choo, J. Kim, Kang, H. K. Kim, J. Paik, and D. Won, "Password-only authenticated three-party key exchange with provable security in the standard model," The Scientific World Journal, 2014.

[10] H. Jeong, D. Won, and S. Kim, "Weaknesses and improvement of secure hash-based strong-password authentication protocol," Journal of Information Science and Engineering, vol. 26, no. 5, September 2010, pp. 1845-1858.

[11] A. K. Das, "Analysis and improvement on an efficient biometric-based remote user authentication scheme using smart cards," Information Security, IET 5.3, 2011, pp. 145-151.

[12] L. Jiping, D. Yaoming, X. Zenggang, and L, Shouyin, "An improved biometric-based user authentication scheme for C/S system," International Journal of Distributed Sensor Networks, 2014.

[13] P. Kocher, J. Jaffe, B. Jun, "Differential power analysis," in Proc. the 19th Annual International Cryptology Conference on Advances in Cryptology, 1999, pp. 388-397.

[14] T. S. Messerges, E. A. Dabbish, and R. H. Sloan, "Examining smart-card security under the threat of power analysis attacks," IEEE Transactions on Computers, 2002, vol. 51, no. 5, pp. 541-552. 
[15] T. S. Messerges, E. A. Dabbish, and R. H. Sloan, "Examining smart-card security under the threat of power analysis attacks," IEEE Transactions on Computers, vol. 51, no. 5, pp. 541-552.

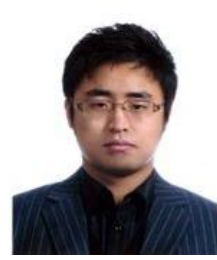

Younsung Choi received the B.S. degree in electrical and computer engineering from Sungkyunkwan University, Korea, in 2006 and the M.S. degree in electrical and computer engineering from Sungkyunkwan University, Korea, in 2007. He is currently undertaking a Ph.D. course on electrical and computer engineering in Sungkyunkwan University. His current research interest is in the area of digital forensic, cyber-crime, cryptography, authentication protocol, and mobile security.

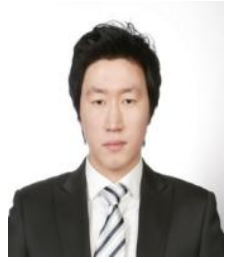

Donghoon Lee received the B.S. degree Computer Science from National Institute for Lifelong Education (NILE), Korea, in 2009 and the M.S. degree in electrical and computer engineering from Sungkyunkwan University, Korea, in 2011. He is currently undertaking a Ph.D. course on electrical and computer engineering in Sungkyunkwan University. His current research interest is in the area of digital forensic, cryptography, forensic, authentication protocol, and mobile security.

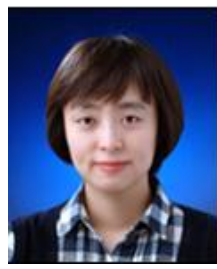

Jiye Kim received the B.S. degree in information engineering from Sungkyunkwan University, Korea, in 1999 and the M.S. degree in computer science Education from Ewha Womans University, Korea, in 2007. He is currently undertaking a Ph.D. course on electrical and computer engineering in Sungkyunkwan University. His current research interest is in the area of cryptography, forensic, authentication protocol, and

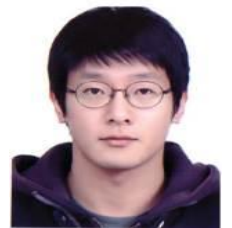

Jaewook Jung received the B.S. degree in electrical and computer engineering from Korea Aerospace University, Korea, in 2010 and the M.S. degree in electrical and computer engineering from Sungkyunkwan University, Korea, in 2012. He is currently undertaking a Ph.D. course on electrical and computer engineering in Sungkyunkwan University. His current research interest is in the area of cryptography, forensic, authentication protocol, and mobile security.

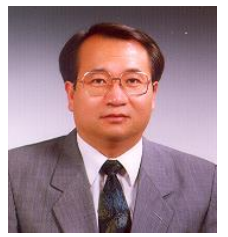

Dongho Won received his B.E., M.E., and Ph.D. from Sungkyunkwan University in 1976, 1978, and 1988 respectively. After working at ETRI (Electronics \& Telecommunications Research Institute) from 1978 to 1980, he joined Sungkyunkwan University in 1982 , where he is currently a professor of the School of Information and Communication Engineering. In the year 2002, he served as the president of KIISC (Korea Institute of Information Security \& Cryptology). He was the program committee chairman of the 8th International Conference on Information Security and Cryptology (ICISC 2005). His research interests are on cryptology and information security. 